\title{
Low-grade fibromyxoid sarcoma of the maxillary sinus
}

\author{
Vladimir Kouckya , David Kalferta ${ }^{\mathrm{a}}$, Daniela Kodetova Novakova ${ }^{\mathrm{b}}$, Jan Plzak
}

\begin{abstract}
Background. Low-grade fibromyxoid sarcoma (LGFMS) is a rare malignant tumor. Moreover, only 3 cases of LGFMS originating from the nasal cavity and/or paranasal sinuses have been published so far.

Methods. Two patients with histologically confirmed LGFMS of the maxillary sinus were primarily treated by open and endoscopic surgery. In one case adjuvant radiotherapy was indicated because of the uncertainty of the surgical margins. Results. Both surgeries were technically demanding and accompanied by significant bleeding from the tumors. Despite the extensive interventions there were no postoperative complications and no significant morbidity of the patients. There is no evidence of the disease 148 and 65 months after the treatment.

Conclusions. Regardless of the excellent post-treatment results, based on our experience, preoperative embolisation of the tumor should be considered prior to the surgical resection.
\end{abstract}

Key words: sarcoma, low-grade fibromyxoid sarcoma, head and neck, maxillary sinus, endoscopic sinus surgery

Received: January 27, 2020; Revised: July 6, 2020; Accepted: July 15, 2020; Available online: July 20, 2020

https://doi.org/10.5507/bp.2020.032

(c) 2021 The Authors; https://creativecommons.org/licenses/by/4.0/

${ }^{a}$ Department of Otorhinolaryngology and Head and Neck Surgery, First Faculty of Medicine, Charles University and Motol University Hospital, V Uvalu 84, 15006, Prague, Czech Republic

${ }^{b}$ Department of Pathology and Molecular Medicine, Second Faculty of Medicine, Charles University and Motol University Hospital, V Uvalu 84, 15006, Prague, Czech Republic

Corresponding author: Vladimir Koucky, e-mail: vladimir.koucky@fnmotol.cz

\section{INTRODUCTION}

Low-grade fibromyxoid sarcoma (LGFMS) is a rare tumor counting less than 400 cases found in literature worldwide. The disease was described by Evans et al. in 1987, who published 2 cases of potentially metastasizing soft tissue tumor with deceptively benign histology, consisting of alternating myxoid and fibrous areas ${ }^{1}$. Typical sites of the occurrence are deep soft tissues of the trunk and extremities. Only around 20 cases of LGFMS in the head and neck have be reported; however, most of them are part of case series studies without specification of the tumor location ${ }^{2}$. To the best of our knowledge, there are only 2 brief reports describing LGFMS in the nasal cavity or paranasal sinuses s.4 $^{3,4}$ and 1 case included in the patient cohort, but not discussed further ${ }^{5}$. We present in detail 2 cases of LGFMS of the maxillary sinus that were diagnosed and treated at our department.

\section{Case 1}

A 57- year-old woman visited our outpatient department for 2 years persisting nasal obstruction on the left side. On the physical examination there was an uncertain finding of a hypertrophy of the mucous membrane of the middle concha and a tissue obturation of the upper part of the left choana. CT scan showed a pathological mass originating from the antrum of the left maxillary sinus and invading the nasal cavity (Fig. 1A). Based on the endoscopic examination and CT scan a malignant tumor was included in the differential diagnosis and the patient was indicated for endoscopy under general anesthesia. During the endoscopy, a greyish vasculated tumor on the lateral side of the nasal cavity was described. We evaluated that endoscopic resection was not possible in a radical fashion. A biopsy was taken but immediately after that severe bleeding from the tumor occurred and had to be controlled by an anterior nasal package. After the first procedure, MRI was performed during hospitalization, clarifying propagation of the tumor into the fossa pterygopalatina. The tumor was nonhomogeneous in signal density, but generally hyposignal (Fig. 1B,C). The acquired histology was described as chondroid chordoma. A decision on radical surgery was made, however, due to the bleeding experience and spreading of the tumor to the pterygopalatine fossa, an open resection through lateral rhinotomy with anterior and posterior ethmoidectomy, sphenoidectomy and resection of medial and posterior wall of the maxillary sinus was performed. Resection of the tumor from the pterygopalatine fossa was accomplished in a piecemeal fashion. There was no postoperative complication. Unexpectedly, a final histological evaluation revealed low-grade fibromyxoid sarcoma. Due to the spread of the disease and uncertainty about surgical margins, adjuvant radiotherapy was indicated at a total dose of $60 \mathrm{~Gy}$ on the locality of the tumor. The patient was followed regularly using endoscopic examination and imaging methods. At the time of writing there has been no evidence of the disease 148 months after the surgery.

\section{Case 2}

A 74-year-old man was referred to our department with a suspicious mucocele of the right maxillary sinus. Nasal obstruction progressing over two months was the only symptom reported by the patient. During endoscopic ex- 

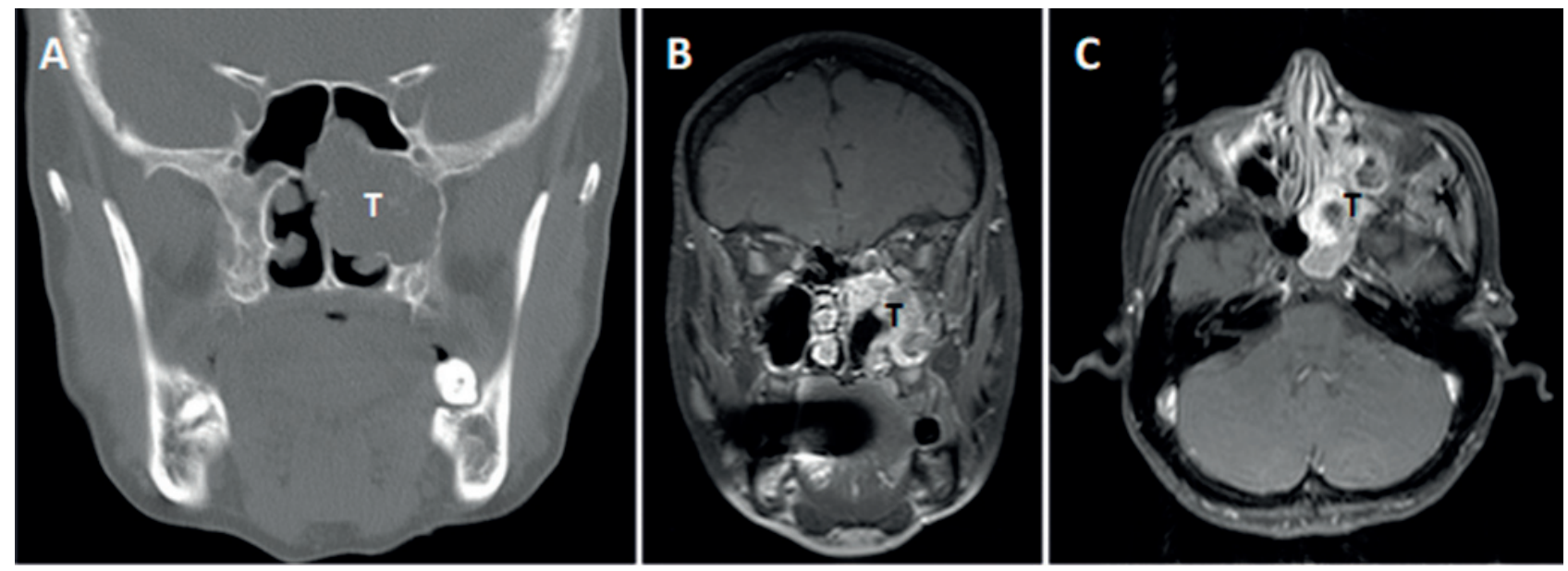

Fig. 1. Preoperative CT and MRI scans of patient 1 show tumor mass in the left maxillary sinus.

A - CT scan, coronar reconstruction, bone window. B, C - MRI of Patient 1 after the endoscopic surgery and taking of biopsy, with necessary application of anterior nasal package.

Coronar (B) and axial (C) reconstructions. Both T1- weighted images. T - tumor
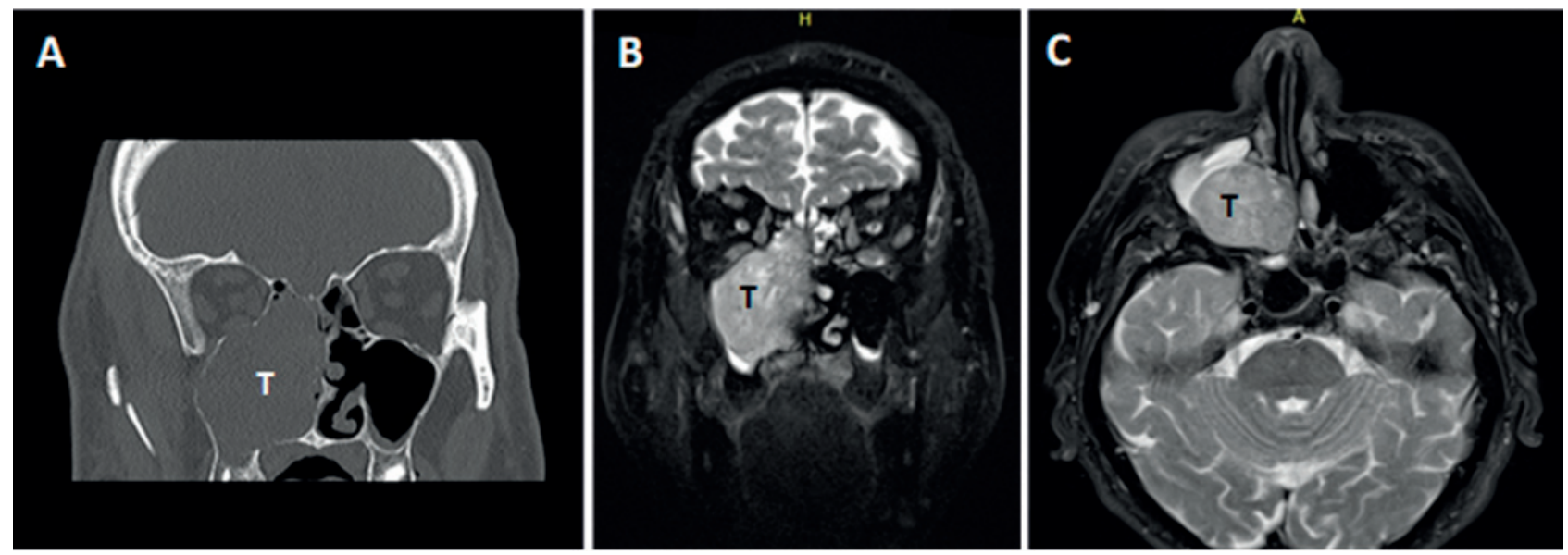

Fig. 2. Preoperative CT nad MRI scans of patient 2 show tumor mass in the right maxillary sinus.

A - Preoperative CT scan, coronar reconstruction, bone window. B, C - Preoperative MRI, coronar (B) and axial (C) reconstructions, both T2- weighted images. T - tumor

amination a bulging of the lateral wall of the nasal cavity covered with an edematous mucous membrane was found. CT showed a homogeneous expansion growing from the right maxillary sinus, invading ethmoidal air cells and dislocating the base of the orbit, but the bony borders of the orbit and maxillary sinus were not disrupted (Fig. 2). MRI was indicated and showed a tumor of approximately $5.5 \mathrm{~cm}$ in size, filling the right maxillary sinus and protruding to the right nasal meatus up to the lamina cribrosa. Nevertheless, no intracranial spread was observed. The tumor was hyposignal on T1 and T2 sequences with nonhomogenous enhancement after contrast application (Fig. 2B,C). The decision was made to perform surgery with perioperative histology evaluation followed by a complete resection. Perioperative histology showed a mesenchymal tumor with low mitotic activity. Given our acquisition of experience with endoscopic endonasal surgery, the resection was performed using endoscopic medial maxillectomy approach. It was a technically difficult four-handed surgery complicated by severe bleeding from the tumor.
Nevertheless, the bleeding was controlled endoscopically and the postoperative course was without any complication. A final histology report described the tumor as containing myxoid and cellular areas with star-shaped cells and rich vascularization. Following wide immunohistochemical testing the final diagnosis was LGFMS (Fig. 3). Despite the larger size of the tumor in comparison to Case 1 , the surgery was radical and no adjuvant treatment was indicated. The patient is kept under surveillance using endoscopic examination and regular CT/ ultrasonography of the chest and peritoneal cavity. At the time of writing, no evidence of the disease has been registered 65 months after the resection.

\section{DISCUSSION}

LGFMS is a soft tissue neoplasm with an unclear incidence, although, a study from western Denmark estimates the incidence is 0.18 per million ${ }^{6}$. The disease preferen- 


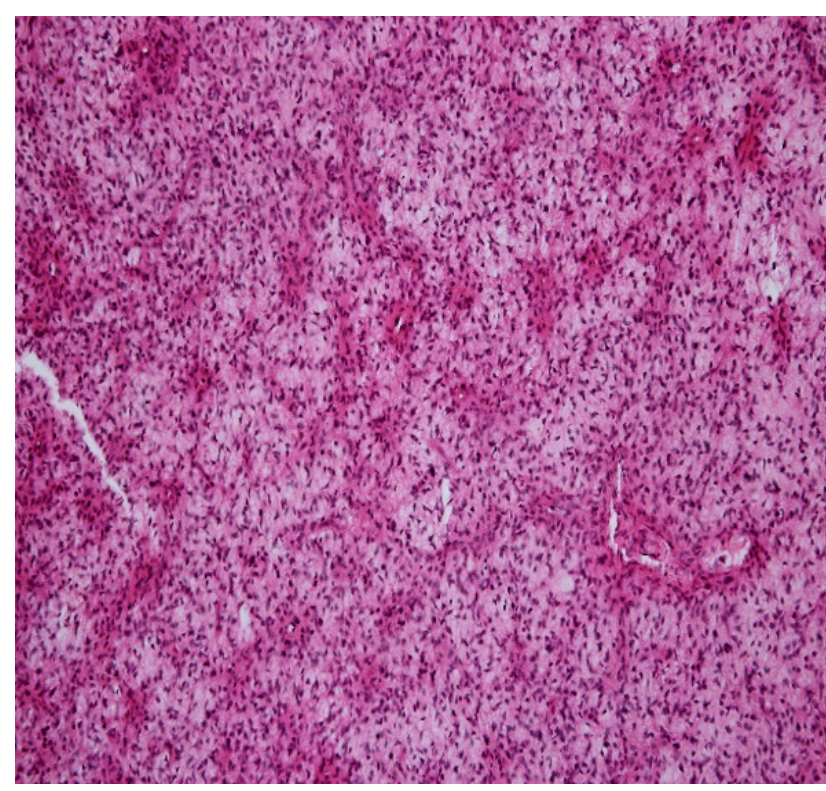

Fig. 3. Microscopic view of the tumor resected from Patient 2, 100x magnification.

tially affects young and middle-aged adults with a median age ranging from 29 to 40 years ${ }^{7}$. Nevertheless, LGFMS can develop at any age including extremely rare cases in infants, such as the case of a 9-year-old boy with LGFMS of the superior turbinate ${ }^{4}$.

The typical symptom is a painless, slow growing mass. The size of the tumor varies from 1 to approximately $19 \mathrm{~cm}$ at the time of the diagnosis ${ }^{5}$. In our cases, tumors were 4 and $5.5 \mathrm{~cm}$ in their greatest diameter. Despite being quite large masses concerning location, probably the slow, noninvasive growth of the tumors caused late and only less serious symptoms. The majority of the tumors are located in the soft tissues of the trunk, lower and upper extremities, but even atypical sites such as heart or thyroid gland are described ${ }^{8,9}$. The clinical course of the disease is unpredictable and insidious. Based on the case series with long term follow up data there is a high potential to develop late local recurrences and distant metastases. In 2011, Evans et al. presented a series of 33 cases where 21 patients had a local recurrence and 15 patients had a distant metastasis. Moreover, the median time to the development of the metastasis was 5 years, ranging from 0 to 42 years $^{7}$. Guillou et al. published a series of 30 patients with long term follow up data, reporting recurrence and metastasis rate to be $20 \%$. Median time to local recurrence and metastasis were 276 and 132 months, respectively ${ }^{10}$. The critical aspect is that $71.4 \%$ of patients with a follow up longer than 7 years developed a metastasis. Tang et al. in 2010 reviewed data of 273 patients with hyalinizing spindle cell tumor or LGFMS, 184 of whom had follow up data available. $29 \%$ of patients had local recurrence and $18 \%$ of patients were diagnosed with a distant metastasis ${ }^{11}$. These data support the necessity for a long term follow up of patients diagnosed with LGFMS.

Radical surgery with wide margins is the treatment of choice. In our cases, the procedures were complicated by severe bleeding from the tumors. Especially in endo- scopic endonasal surgery, bleeding is the main enemy. The reports from Manes et al. and Varsak et al. do not mention excessive bleeding despite the use of endoscopic approach. However, both the cases were much younger patients with significantly smaller tumor size and less prominent spread to the upper and dorsal parts of the nasal cavity, based on the presented MRI. Given our experience, preoperative embolisation of LGFMS in this location should be considered.

On histological examination, LGFMS shows bland looking spindle cell proliferation in a swirling, whorled growth pattern. Alternating myxoid and fibrous zones are characteristic. Immunohistochemically there is no specific marker for LGFMS, yet the tumor shows typically strong and diffuse cytoplasmic positivity to MUC4 (ref. ${ }^{12}$ ). It is a transmembrane glycoprotein expressed on the epithelial surfaces of many organ systems and on some tumors such as pancreatic, biliary duct or lung adenocarcinomas. Its function is associated with phosphorylation of Erb2b tyrosinekinase that plays an important role in the proliferation and differentiation of cells. A characteristic genetic feature is balanced translocation $\mathrm{t}(7 ; 16)$ (q34; p11), that leads to fusion of the FUS and CREB3L2 genes ${ }^{13}$. In a minority of cases there can be a variant fusion FUSCREB3L1 or EWSR1-CREB3L1 ( ref. $^{14}$ ).

Based on a clinical examination, imaging methods and immunohistochemistry, the differential diagnosis is quite large. Pathologic conditions that can show similar patterns are myxofibrosarcoma, dermatofibrosarcoma, fibromatosis, myxoid neurofibroma or sclerosing epitheloid fibrosarcoma (SEF). Moreover, SEF is positive for MUC4 up to $78 \%$ (ref. ${ }^{15}$ ). There is also a tumor variant called hybrid SEF-LGFMS, that contains areas indistinguishable from LGFMS and shows $100 \%$ positivity for MUC4 staining.

\section{CONCLUSIONS}

LGFMS is a rare disease that even less frequently appears in the head and neck. Radical surgery is the primary treatment option although in head and neck there is a risk of serious morbidity and esthetic defect. In view of this fact, the endoscopic approach should be applied when possible. Nevertheless, endoscopic surgery can be a challenging procedure due to the possible extensive bleeding from the tumor as happened in our cases. Based on these experiences, preoperative embolisation of the tumor should be considered. However, even after successful radical surgery, prolonged follow up is necessary due to the metastasizing capacity of the tumor and the possibility of late recurrences.

Acknowledgements: We would like to thank Dr. Jitka Hroboňová for her participation in the treatment of the reported patients. The study was funded by the Czech Science Foundation, grant No. 18-03978S.

Author contributions: VK: data collection and manuscript writing; DK: supervised manuscript preparation and performed critical reading; DKN: performed histopathology 
evaluation and provided microscopic images of the tumor; JP: managed patient treatment and supervised manuscript preparation.

Conflict of interest statement: The authors state that there are no conflicts of interest regarding the publication of this article.

\section{REFERENCES}

1. Evans HL. Low-grade fibromyxoid sarcoma. A report of two metastasizing neoplasms having a deceptively benign appearance. Am J Clin Pathol 1987;88:615-19.

2. Cowan ML, Thompson LD, Leon ME, Bishop JA. Low-Grade Fibromyxoid Sarcoma of the Head and Neck: A Clinicopathologic Series and Review of the Literature. Head Neck Pathol 2016;10:161-6.

3. Manes RP, Lemeshev Y, Batra PS. Pathology quiz case 2. Low-grade fibromyxoid sarcoma (LGFMS). Arch Otolaryngol Head Neck Surg 2011;137:199:201-2.

4. Varsak YK, Arbag H, Yesildemir HS, Esen H. Low-grade fibromyxoid sarcoma of superior turbinate in a pediatric patient. J Craniofac Surg 2015;26:962-4

5. Hwang S, Kelliher E, Hameed M. Imaging features of low-grade fibromyxoid sarcoma (Evans tumor). Skeletal Radiol 2012;41:1263-72.

6. Maretty-Nielsen K, Baerentzen S, Keller J, Dyrop HB, Safwat A. Low-Grade Fibromyxoid Sarcoma: Incidence, Treatment Strategy of Metastases, and Clinical Significance of the FUS Gene. Sarcoma 2013;2013:256280.

7. Evans HL. Low-grade fibromyxoid sarcoma: a clinicopathologic study of 33 cases with long-term follow-up. Am J Surg Pathol 2011;35:1450-62.

8. Ferlosio A, Doldo E, Polisca P, Orlandi A. Low-grade fibromyxoid sarcoma: an unusual cardiac location. Cardiovasc Pathol 2013;22:e1517.

9. Dong W, Zhang H. Low-grade fibromyxoid sarcoma of the thyroid: a case report. Ann Acad Med Singapore 2013;42:55-6.

10. Guillou L, Benhattar J, Gengler C, Gallagher G, Ranchere-Vince $D$, Collin F, Terrier P, Terrier-Lacombe MJ, Leroux A, Marques B, Aubain Somerhausen Nde S, Keslair F, Pedeutour F, Coindre JM. Translocation-positive low-grade fibromyxoid sarcoma: clinicopathologic and molecular analysis of a series expanding the morphologic spectrum and suggesting potential relationship to sclerosing epithelioid fibrosarcoma: a study from the French Sarcoma Group. Am J Surg Pathol 2007;31:1387-402.

11. Tang Z, Zhou ZH, Lv CT, Qin LY, Wang Y, Tian G, Luo XL, Zhu Q, Xu $X G$. Low-grade fibromyxoid sarcoma: clinical study and case report. J Oral Maxillofac Surg 2010;68:873-84.

12. Doyle LA, Moller E, Dal Cin P, Fletcher CD, Mertens F, Hornick JL. MUC4 is a highly sensitive and specific marker for low-grade fibromyxoid sarcoma. Am J Surg Pathol 2011;35:733-41.

13. Panagopoulos I, Storlazzi CT, Fletcher CD, Fletcher JA, Nascimento $A$, Domanski HA, Wejde J, Brosjo O, Rydholm A, Isaksson M, Mandahl N, Mertens F. The chimeric FUS/CREB3/2 gene is specific for low-grade fibromyxoid sarcoma. Genes Chromosomes Cancer 2004;40:218-28

14. Lau PP, Lui PC, Lau GT, Yau DT, Cheung ET, Chan JK. EWSR1-CREB3L1 gene fusion: a novel alternative molecular aberration of low-grade fibromyxoid sarcoma. Am J Surg Pathol 2013;37:734-8.

15. Doyle LA, Wang WL, Dal Cin P, Lopez-Terrada D, Mertens F, Lazar AJ, Fletcher CD, Hornick JL. MUC4 is a sensitive and extremely useful marker for sclerosing epithelioid fibrosarcoma: association with FUS gene rearrangement. Am J Surg Pathol 2012;36:1444-51. 